\title{
The role of $\mathrm{COX}-2$ in rectal cancer treated with preoperative radiotherapy
}

\author{
Hanifa Bouzourene • Pu Yan • Dominique Sandmeier • \\ Abderrahim Zouhair • Maurice Matter • \\ Henri Vuilleumier • Philippe Coucke
}

Received: 4 February 2008 /Accepted: 9 March 2008 / Published online: 5 April 2008

(C) Springer-Verlag 2008

\begin{abstract}
Radiotherapy is one of the principal modalities of rectal cancer treatment, and the ability to predict radio resistance could potentially improve survival through a targeted treatment approach. Cyclooxygenase-2 (COX-2) may protect against damage by irradiation that would justify the use of COX-2 inhibitors. The purpose of this study was to investigate the potential role of COX-2 in tumor response and outcome of patients with rectal cancer treated preoperatively with radiotherapy. Using immunohistochemistry, we examined COX-2 expression in 88 surgical specimens of rectal cancer treated preoperatively and in 26 pretherapeutic biopsies. We tested whether COX2 expression was correlated with clinico-pathologic parameters and with survival and local recurrence. COX-2 was expressed in $50 \%$ of the pretherapeutic tumor biopsies and in $88.6 \%$ of post-irradiated surgical samples. COX-2 expression was correlated only with enhanced tumor
\end{abstract}

H. Bouzourene $(\bowtie) \cdot$ P. Yan $\cdot$ D. Sandmeier

Institute of Pathology, Centre Hospitalier Universitaire Vaudois, Bugnon 25, CH 1011 Lausanne, Switzerland

e-mail: Hanifa.Bouzourene@chuv.ch

A. Zouhair

Department of Radiooncology,

Centre Hospitalier Universitaire Vaudois,

CH 1011 Lausanne, Switzerland

M. Matter $\cdot$ H. Vuilleumier

Department of Surgery, Centre Hospitalier Universitaire Vaudois,

CH 1011 Lausanne, Switzerland

P. Coucke

Department of Radiation-Oncology,

Hôpital Maisonneuve-Rosemont,

5415, Boulevard de l'Assomption,

Montréal, QC, Canada H1T 2M4 inflammation $(p=0.03)$ and with tumor volume exceeding $30 \mathrm{cc}(p=0.05)$. COX-2 was not significantly correlated with patient survival, but none of the patients with COX-2 negative tumors did recur locally, whereas $80 \%$ of patients with local recurrences have COX-2 positive tumors. We conclude that COX-2 expression is overexpressed in the majority of rectal cancers treated with radiotherapy and likely plays a role in local relapse.

Keywords Radiotherapy Rectal cancer

Cyclooxygenase- $2 \cdot$ Survival $\cdot$ Recurrence

\section{Introduction}

Nowadays, many therapeutic procedures are available for locally advanced rectal cancer (LARC). However, except the fact that surgery with total mesorectal excision is the mainstay of treatment $[11-13,16,30]$, there is no consensus amongst the published literature about what should be the "standard" treatment and the primary endpoint in rectal cancer. Studies with systemic treatment do not show an impact of chemotherapy on overall survival or progressionfree survival $[1,10]$. In Europe, in contrast to the United States and Canada, a preoperative radiotherapy has been considered as the preferred treatment option for LARC to reduce the incidence of local recurrence $[17,38]$.

In addition to the problem of the best choice of a standard treatment, the therapeutic efficacy is often hindered by tumor resistance and especially tumor radio resistance. Recent studies indicate that COX-2, an enzyme involved in the metabolic conversion of arachidonic acid to prostaglandins, plays an important role in tumor growth and spread of established tumors by affecting mitogenesis, cellular adhesion, immune surveillance, apoptosis, and 
angiogenesis $[6,21,24,34,44,41,46]$. Several studies have shown that COX-2 expression is elevated in human colorectal neoplasia as compared to normal mucosa, thus making COX-2 a potential target for chemoprevention [7, 15, 42]. A possible relationship between COX-2 expression in colorectal cancer tumors and patient survival has been investigated but nowadays data are inconclusive $[8,20,22$, $28,33,40,48]$. Because COX-2 may also be involved in tumor radio resistance, targeting of COX-2 or downstream products (prostaglandins) may eventually improve tumor response to radiotherapy $[25,26]$. In vitro and in vivo studies have shown that treatment with selective COX-2 inhibitors significantly enhances tumor response to radiation without appreciably affecting normal tissue radio response $[19,23,26,29]$. However, evidence of enhanced tumor response to radiation is less obvious in human models.

The purpose of this study was to investigate the relationship between the radiation resistance and the level of COX-2 on a retrospective series of patients with locally advanced rectal cancer (LARC) treated exclusively with radiotherapy. We therefore investigated COX-2 expression in pretherapeutic biopsies and surgical specimens and we tried to correlate it with histological response and clinical outcome.

\section{Materials and methods}

\section{Patients}

Between 1993 and 2002, 279 patients with locally advanced rectal cancer were included in a multicenter phase II trial on preoperative hyperfractionated and accelerated radiotherapy approved by the Human Investigations Committee of the radiation oncology centers [5]. Only 104 patients treated in our institution and already selected for a previously reported study were considered for this analysis [2].

Patients were eligible if they presented at preoperative work-up with cT3-cT4, whatever the N-stage, or cT1-cT2 with radiological evidence of positive lymph nodes. All patients were treated on protocol after obtaining informed consent for treatment. The age of the patients ranged from 28 to 85 (median, 63 years) and the sex ratio was 1.2 (female/ male). Before initiation of the treatment, all patients underwent complete clinical examination, endoscopic biopsy, blood count, assessment of renal and hepatic function, and CEA determination. Distant metastatic disease was excluded by chest X-ray and abdominal ultrasound or computed tomography (CT scan). Assessment of the local extension of the tumor was done by digital rectal examination, rectal ultrasound, and/ or CT scan. All patients were irradiated according to the same protocol [5]. The decision concerning the surgical technique, mainly abdominoperineal resection (APR) or low anterior resection, is left to the individual surgeons. The protocol stipulates that a total mesorectal excision with a sharp dissection is "the surgical standard" for those rectal tumors.

\section{Biopsy specimens}

Only 26 patients had pretherapeutic biopsies that were available for immunohistochemistry. Biopsies had been fixed in $10 \%$ neutral formalin for $12-24 \mathrm{~h}$ at room temperature. In the remainder cases, the tumor samples had been exhausted during previous studies and were no more present in the paraffin blocks or immunostaining failed because the material had been fixed in sublimated formol.

\section{Macroscopical assessment of surgical specimens}

The surgical specimens were fixed in $10 \%$ buffered neutral formalin for $24 \mathrm{~h}$. The whole tumor and attached mesorectum were serially sliced at 3- to 4-mm intervals perpendicular to the longitudinal axis of the rectum, which allowed macroscopical identification of the areas of deepest invasion.

\section{Histological assessment of surgical specimens}

All irradiated rectal tumors were retrospectively reanalyzed (H.B). The tumors were classified according to the WHO classification of tumors of the digestive system [47] and staged according to the TNM classification [35]. Tumor downstaging was obtained by comparison of the clinical preoperative and postoperative pathological tumor staging. Tumor volume was calculated on the length and width evaluated on the macroscopical examination and on the tumor thickness assessed on slides. Tumor inflammation, tumor necrosis, and tumor regression were graded as previously described [2]. Tumor inflammatory reaction (mononuclear and granular cells) was graded as 1 when absent or mild, 2 when moderate, and 3 when extensive. Tumor necrosis was graded as 1 when absent or less than $25 \%$ of the tumor mass, 2 when it represented $25-50 \%$ of the tumor mass, 3 when it represented $50-75 \%$ of the tumor mass, and 4 when it represented $75 \%$ of the tumor mass. Tumor regression was graded in five grades, based on the presence of residual tumor cells and the extent of fibrosis. Grade 1 was defined as sterilization of the tumor, grade 2 by the presence of rare residual cancer cells scattered through the fibrosis, grade 3 by more residual tumor cells but fibrosis still predominates, grade 4 by more residual cancer cells than fibrosis, and grade 5 when the tumor shows no signs of regression. Vascular invasion was also recorded.

\section{COX-2 immunohistochemistry}

Four-micrometer-thick tissue sections were deparaffinized in xylene, rehydrated, treated with $0.3 \% \mathrm{H}_{2} \mathrm{O}_{2}$ in methanol 
for $30 \mathrm{~min}$ to block endogenous peroxidase activity. The antigen retrieval was done using a pressure cooker. After preheating 11 of $0.01 \mathrm{M}$ sodium citrate buffer, pH6.0 until boiling in a stainless steel pressure cooker, the sections were heated for $2 \mathrm{~min}$, during which the pressure cooker reached operating temperature. Subsequently, the pressure cooker was removed from the heat source and placed under running cold water with the lid on to cool it down. The slides were placed in a container with running cold water and cooling continued for another $10 \mathrm{~min}$. The sections were subsequently rinsed in phosphate-buffered saline (PBS) and conditioned with 10\% normal goat serum for $30 \mathrm{~min}$ at room temperature (RT) and incubated with antiCOX-2 monoclonal antibody (Cayman Chemical, Ann Arbor, MI, USA) diluted at 1:200 in PBS with $0.1 \mathrm{M} \mathrm{NaCl}$ at RT for $2 \mathrm{~h}$. After rinsing in PBS, the slides were incubated with DAKO EnVision ${ }^{\mathrm{TM}}$ (mouse) for $30 \mathrm{~min}$ at RT. After the slides were rinsed in PBS, peroxidase was revealed by immersion in diaminobenzidine (DAB; DAKO, Carpentiria, CA, USA) according to the manufacture's instructions and the sections were counterstained with hematoxylin and mounted for microscopic examination. The negative control was performed by using COX-2 blocking peptide diluted at 1:50 according to the manufacture's instructions (Cayman Chemical). Immunohistochemical expression of COX-2 was evaluated semiquantitatively by two observers using a double-headed microscope (H.B, P.Y) on blinded sections. There was no staining on the negative control slide. Staining for COX-2 in the normal rectal mucosa was used as a positive control. Absorptive epithelial cells bordering the luminal surface and especially basal part of the crypts showed COX-2 positivity, generally localized in the paranuclear region and cell membranes, less frequently in the nuclei. If no crypt staining was obtained after two attempts, the case was eliminated from the study on technical grounds. In tumor cells, COX-2 immunoreactivity was observed in the cytoplasm and/or in the nuclei (Fig. 1). The spindle-shaped stromal cells, intermingled with neoplastic glands, sometimes showed staining for COX-2. All cases were scored as following: score $0:<5 \%$ of positive cells; score 1: 5-30\% of positive cells; score 2: $30-60 \%$ of positive cells; score 3: $>60 \%$ of positive cells. Tumor cases with COX-2 score 0 were considered as negative.

\section{Statistical analysis and follow-up}

All patients were followed for local recurrence and distant metastasis every 6 months for the first 2 years and every year thereafter. A physical examination, serum carcinoembryonic antigen (CEA) assay, chest X-ray, abdominal ultrasound or CT scan, and a CT scan of the pelvis were included in the follow-up procedure. The events were death (all causes of death included) for overall survival (OS), distant metastases or locoregional relapse or death for disease-free survival (DFS), and local or locoregional relapse for local control (patients who died without local or locoregional relapse were censored at time of death), respectively.

All statistical analyses were conducted using the JUMP software (SAS, Cary, NC). A $p$ value $<0.05$ was considered statistically significant. In univariate and multivariate analysis, OS, DFS, and local control were used as the end points. In univariate analysis survival curves were estimated according to the Kaplan-Meier method for the following variables: age, sex, $\mathrm{pT}, \mathrm{pN}$, tumor differentiation, tumor inflammation, tumor necrosis, tumor regression, tumor volume, and vascular invasion. The significance of their difference was estimated by the log-rank test. To increase the number of patients per group, the categories of the different pathological variables were also combined for these analyses, i.e., pT1-2 vs pT3-4; well and moderately differentiated adenocarcinomas vs poorly differentiated and mucinous carcinomas; absence or mild necrosis (score 1) vs moderate to extensive necrosis (scores 2-4); absence or mild inflammation (score 1) vs moderate to marked inflammation (scores 2 and 3); responder group (tumor regression grades 2-4) vs non-responder group (tumor regression grade 5).

Multivariate survival analysis according to the Cox's proportional hazards models was constructed by backward elimination of the following variables: age, sex, $\mathrm{pT}, \mathrm{pN}$, tumor differentiation, tumor inflammation, tumor necrosis, tumor regression, and vascular invasion.

\section{Results}

Pathological findings

Eighty-eight out of 104 patients with rectal cancer were selected for the COX-2 study. The remainder cases were excluded because the tumour was sterilized (tumor regression grade 1) or for technical reasons. There were $20 \mathrm{pT} 2$ tumors (23\%), 57 pT3 (65\%), and 11 pT4 (12\%). Tumor downstaging obtained after comparison of the clinical and pathological stages was observed in $44 \%$ of the cases. Regional lymph node metastases were found in $59 \%$ of patients. Of the tumors, $25 \%$ were well-differentiated adenocarcinomas, $42 \%$ were moderately differentiated adenocarcinomas, $10 \%$ were poorly differentiated adenocarcinomas, and $25 \%$ were mucinous carcinomas. Partial tumor regression (G2-4) was noted in $80 \%(\mathrm{G} 2=16 \%$; $\mathrm{G} 3=39 \%$; G4 $=25 \%$ ) and absence of regression (G5) was noted in $20 \%$ of the tumors. Tumor necrosis was absent or mild (score 1 ) in $66 \%$ of the tumors, moderate to extensive 
Fig. 1 Low (a) and high magnification (b) of COX-2 expression in a rectal adenocarcinoma treated preoperatively with radiotherapy

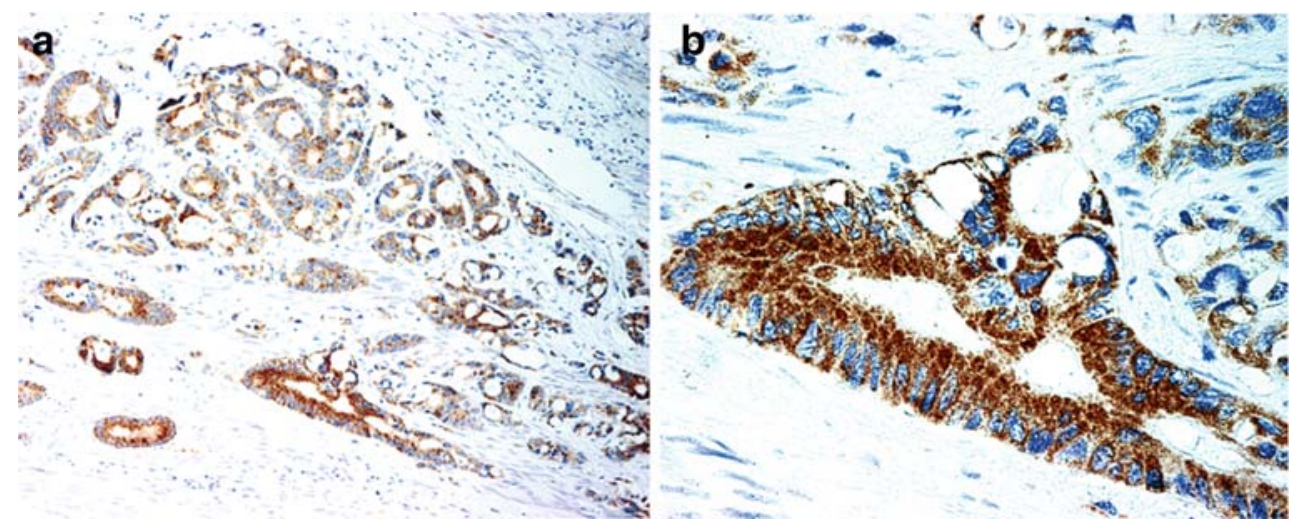

in $34 \%$ (scores $2-4$ ) of the tumors. Tumors showed a mild or no inflammation (score 1) in $30 \%$ of cases, a moderate to marked inflammation in $70 \%$ (score 2 and 3 ) of cases. Vascular invasion was present in 29 patients.

Survival and local control

During follow-up (median=42 months), 24 patients developed distant metastasis and eight patients a local recurrence. Thirty-nine patients died, 29 of whom from rectal cancer.

The median OS was 53 months. The median actuarial OS was significantly lower in patients with lymph node metastases $(p=0.005, \log$ rank test) and for those patients presenting tumors with vascular invasion $(p=0.003)$. There was no difference in OS between responders (G2-4) compared to non-responders (G5).

The median DFS was 46 months. The median actuarial DFS was significantly lower in patients with advanced $\mathrm{pT}$ stages (pT3-4) $(p=0.03$, log rank test) and with lymph node metastases $(p=0.0008, \log$ rank test). The DFS was longer in responders than in non responders $(p=0.03, \log$ rank test). Moreover, a significant correlation was found between the different grades of tumor regression and DFS ( $p=0.04, \log$ rank test).

The actuarial locoregional recurrence rates at 2 and 5 years were $7.5 \%$ and $11.4 \%$, respectively. There was a trend for a better local control in responders as compared to non-responders $(p=0.10, \log$ rank test).

Cyclooxygenase-2 immunostaining and pathologic analysis

By immunohistochemistry COX-2 was found to be absent in $13(50 \%)$ out of 26 tumor biopsies taken before treatment (score 0). In tumor biopsies expressing COX-2, four cases were scored 1, four cases were scored 2, and five cases were scored 3.

In 88 surgical specimens, ten tumors $(11 \%)$ did not express COX-2 (score 0). Of 78 COX-2 positive tumors $(89 \%), 38(44 \%)$ had a mild (score 1), $25(28 \%)$ a moderate (score 2), and $15(17 \%$ ) a marked (score 3) COX-2 expression. The distribution of COX-2 expression and pathologic parameters is summarized in the Table 1. When comparing COX-2 expression in biopsies and surgical specimens we found that among 13 patients with negative tumor biopsies, ten $(78 \%)$ became COX-2 positive in the surgical specimens with a scoring of expression varying from 1 to 3 , whereas only three cases remained negative after irradiation. Among the cases with positive COX-2 in tumor biopsies, two had a higher score of expression, seven had the same score of expression, whereas four cases had a lower score of expression in the irradiated tumors.

COX-2 expression was not correlated with any of the following parameters: sex, age, tumor downstaging, pT, pN, tumor regression, tumor necrosis, vascular invasion. We found a trend of correlation between COX-2 and tumor differentiation $(p=0.087)$. COX-2 was correlated with moderate and marked tumor inflammation $(p=0.03)$ and with tumor volume exceeding $30 \mathrm{cc}(p=0.05)$. Grouping according to level of expression of COX-2 with any of the tested pathological variables did not modify the conclusions of the present analysis.

We were not able to demonstrate any significant correlation between the expression level of COX-2 and OS and DFS. However, it is noteworthy that none of the patients having tumor without COX-2 expression did recur locally $(0 / 10)$, in contrast to eight out of ten recurrences $(80 \%)$ in 78 patients with tumor characterized by a COX-2 expression. It might well be that the number of local events is too small in the present series to allow us to highlight a significant difference.

\section{Discussion}

Colorectal cancer is a significant cause of morbidity and mortality in Western populations. Rectal cancer if treated by surgery alone is characterized by a high incidence of local recurrence (20 to $70 \%$ ), regional lymph node and distant metastases [17, 38]. Until now, there is no consensus 
Table 1 Correlations between COX-2 expression and histological parameters in 88 surgical specimens of rectal cancer treated with preoperative radiotherapy

\begin{tabular}{|c|c|c|c|c|c|c|c|c|c|}
\hline \multirow{3}{*}{ Scores of COX-2 expression } & \multicolumn{8}{|c|}{ COX- 2 expression } & \multirow[t]{3}{*}{$p$ value } \\
\hline & \multicolumn{2}{|l|}{0} & \multicolumn{2}{|l|}{1} & \multicolumn{2}{|l|}{2} & \multicolumn{2}{|l|}{3} & \\
\hline & $N$ & $\%$ & $N$ & $\%$ & $N$ & $\%$ & $N$ & $\%$ & \\
\hline Pathological staging & & & & & & & & & NS \\
\hline pT2 & 4 & 4 & 7 & 8 & 4 & 5 & 5 & 6 & \\
\hline pT3 & 5 & 6 & 20 & 23 & 19 & 21 & 13 & 15 & \\
\hline pT4 & 1 & 1 & 5 & 6 & 3 & 3 & 2 & 2 & \\
\hline pNo & 6 & 7 & 10 & 11 & 14 & 16 & 10 & 11 & \\
\hline $\mathrm{pN} 1$ & 4 & 5 & 21 & 24 & 10 & 11 & 10 & 11 & \\
\hline Tumor differentiation & & & & & & & & & 0.087 \\
\hline Well differentiated & 2 & 2 & 8 & 9 & 3 & 3 & 7 & 8 & \\
\hline Moderately differentiated & 6 & 7 & 9 & 10 & 13 & 15 & 9 & 10 & \\
\hline Poorly differentiated & 0 & 0 & 5 & 6 & 2 & 2 & 2 & 2 & \\
\hline Mucinous & 2 & 2 & 9 & 10 & 9 & 10 & 2 & 2 & \\
\hline Tumor regression & & & & & & & & & NS \\
\hline Partial (grades 2-4) & 9 & 10 & 23 & 26 & 20 & 23 & 18 & 20 & \\
\hline Absent (grade 5) & 1 & 1 & 9 & 10 & 3 & 3 & 5 & 6 & \\
\hline \multicolumn{10}{|l|}{ Tumor inflammation } \\
\hline Mild (score 1) & 4 & 5 & 10 & 11 & 6 & 7 & 6 & 7 & \\
\hline Moderate to marked (score 2,3 ) & 6 & 7 & 23 & 26 & 20 & 23 & 13 & 15 & 0.03 \\
\hline Tumor necrosis & & & & & & & & & NS \\
\hline Absent or mild (score 1) & 10 & 11 & 30 & 34 & 22 & 25 & 16 & 18 & \\
\hline Moderate to marked (scores 2-4) & 0 & 0 & 3 & 3 & 4 & 4 & 3 & 3 & \\
\hline Vascular invasion & & & & & & & & & NS \\
\hline Present & 5 & 6 & 9 & 10 & 10 & 11 & 5 & 6 & \\
\hline Absent & 5 & 6 & 22 & 25 & 17 & 19 & 15 & 17 & \\
\hline
\end{tabular}

NS non significant

among the published literature about what should be the "standard" treatment and the primary endpoint in rectal cancer (survival, disease-free survival, local control, sphincter sparing surgery or quality of life). However, neoadjuvant preoperative radiotherapy has been shown to induce tumor regression significantly, improving local control and overall survival $[2,5,32]$. Nevertheless, the tumor response, which is different from one patient to another, is probably limited by many genetic factors. Among those factors, the COX-2 appears to play an important role. Cyclooxygenase is a key enzyme that catalyses the conversion of prostaglandins and other eicosanoids from arachidonic acid. COX-2 is induced during pathologic conditions and is often overexpressed in premalignant lesions and malignant tumors $[3,6,24,43$, 44]. This enzyme is linked to carcinogenesis, maintenance of progressive tumor growth, and facilitation of metastatic spread $[4,41]$. Several studies have shown that COX-2 expression is elevated in human adenomas and colon cancers $[7,31,33]$.

Data about the relationship between COX-2 expression and patient survival in colorectal cancer are inconclusive [8, 15, 20, 22, 28, 33, 36, 40, 48]. However, COX-2 protein expression has become the focus of active investigation because of its possible implication in tumor radio resistance. Indeed, it was reported that COX2 is upregulated by radiation, inducing radio resistance $[25,26,29]$. Preclinical studies have shown that treatment with selective COX-2 inhibitors significantly enhances tumor response to radiation without appreciably affecting normal tissue radio response $[19,26]$. However, if in vitro and in vivo results support this hypothesis, the data available from human studies are scarce. In oral squamous cell carcinoma [39] and in laryngeal cancer [27] elevated COX-2 expression has been associated with radio resistance and diminished survival. Expression of COX-2 was also correlated with poor prognosis in squamous cell carcinoma of the uterine cervix treated with radiation therapy $[9,18]$.

In the present study, we investigated by immunohistochemistry the expression of COX-2 in 88 surgical specimens from a cohort of patients with rectal cancer treated preoperatively with radiotherapy and attempted to correlate this with pathologic parameters and clinical outcome. We also investigated the expression of COX-2 before neoadjuvant radiotherapy in a subset of rectal cancers with available tumor biopsies. COX-2 expression was present in 
$50 \%$ of pretherapeutic tumor biopsies and $89 \%$ of irradiated tumors. We found also that the large majority of negative tumor biopsies expressed COX-2 protein after radiotherapy and that a subset of positive tumor biopsies has an increased expression. This finding support the fact that, at least in a subgroup of rectal cancer, COX-2 is upregulated by radiation as already reported in some cancer cell lines [37]. However, this is in partial contradiction with a recent study that compared COX-2 expression in pretherapeutical rectal carcinoma biopsies and posttherapeutical surgical specimen and which found that COX-2 expression is present in nearly all pretherapeutical biopsies although there is an increase of the intensity of COX-2 expression in one third of posttherapeutical rectal cancer [45].

In the present analysis, COX-2 expression in post irradiated tumors was not correlated with any of the tested pathologic parameters in particular with tumor regression and tumor downstaging. We found that $\mathrm{COX}-2$ was correlated with tumor inflammation which is in accordance with the fact that COX-2 is known to be induced by proinflammatory cytokines and that COX-2 is upregulated at sites of inflammation [14]. We also found that COX2 was more frequently expressed when tumor volume exceeds $30 \mathrm{cc}$. We were not able to provide an explanation for this finding especially as we did not find any correlation with tumor regression or tumor downstaging.

More importantly, we found that COX-2 expression is not a significant prognostic factor neither for overall patient survival nor for DFS in irradiated rectal carcinomas. These data are in line with one published study on human rectal cancer pretreated with chemoradiotherapy [45] but are not in accordance with the results reported in studies on squamous cell carcinoma of head and neck and cervix treated with radiotherapy alone or radiochemotherapy $[39,27,9,18]$.

Nevertheless, we found that there was a trend toward increased local failure in patients with treated tumors expressing COX-2. In contrast, all the patients with COX2 negative tumors were free of local recurrence. The difference was not statistically significant, probably due to the small number of recurrences overall. However, testing of this hypothesis within the frame of a randomized trial could be interesting.

\section{Conclusion}

This study shows that radiotherapy induces an expression of COX-2 in the majority of rectal cancer patients and is correlated with tumor inflammation. COX-2 expression is not associated with rectal cancer survival and tumor response, but a possible association with local recurrence remains to be evaluated in a larger cohort of patients with rectal cancer treated by preoperative radiotherapy.
Acknowledgment This work was supported by grant from Fonds national Suisse de la recherche scientifique (FNRS 3100-63835).

Conflict of interest statement We declare that we have no conflict of interest.

\section{References}

1. Bosset JF, Calais G, Mineur L, Maingon P, Radosevic-Jelic L, Daban A, Bardet E, Beny A, Briffaux A, Collette L (2005) Enhanced tumorocidal effect of chemotherapy with preoperative radiotherapy for rectal cancer: preliminary results-EORTC 22921. $\mathrm{J}$ Clin Oncol 23:5620-5627

2. Bouzourene H, Bosman FT, Seelentag W, Matter M, Coucke $P$ (2002) Importance of tumor regression assessment in predicting the outcome in patients with locally advanced rectal carcinoma treated with preoperative radiotherapy. Cancer 94:1121-1130

3. Chan G, Boyle JO, Yang EK, Zhang F, Sacks PG, Shah JP, Edelstein D, Soslow RA, Koki AT, Woerner BM, Masferrer JL, Dannenberg AJ (1999) Cyclooxigenase-2 expression is upregulated in squamous cell carcinoma of the head and neck. Cancer Res 5:991-994

4. Coffey RJ, Hawkey CJ, Damstrup L, Graves-Deal R, Daniel VC, Dempsey PJ, Chinery R, Kirkland SC, DuBois RN, Jetton TL, Morrow JD (1997) Epidermal growth factor receptor activation induces nuclear targeting of cyclooxygenase-2, basolateral release of prostaglandins, and mitogenesis in polarizing colon cancer cells. Proc Natl Acad Sci U S A 94:657-662

5. Coucke P, Notter M, Stamm B, Matter M, Fasolini F, Schlumpf R, Matzinger O, Bouzourene H (2006) Preoperative hyperfractionated acccelerated radiotherapy (HART) in locally advanced rectal cancer (LARC) immediately followed by surgery. A prospective phase II trial. Radiother Oncol 79:52-58

6. Dubois RN, Abramson SB, Crofford L, Gupta RA, Simon LS, Van De Putte LB, Lipsky PE (1998) Cyclooxygenase in biology and disease. FASEB J 12:1063-1073

7. Eberhart CE, Coffey RJ, Radhika A, Giardiello FM, Ferrenbach S, Dubois RN (1994) Up-regulation of cyclooxigenase-2 gene expression in human colorectal adenomas and adenocarcinomas. Gastroenterology 107:1183-1188

8. Fux R, Schwab M, Thon KP, Gleiter CH, Fritz P (2005) Cycloooxygenase-2 expression in human colorectal cancer is unrelated to overall patient survival. Clin Cancer Res 11:4754-4760

9. Gaffney DK, Holden J, Davis M, Zempolich K, Murphy KJ, Dodson M (2001) Elevated cyclooxygenase-2 expression correlates with diminished survival in carcinoma of the cervix treated with radiotherapy. Int $\mathrm{J}$ Radiat Oncol Biol Phys 49:1213-1217

10. Gérard JP, Conroy T, Bonnetain F, Bouché O, Chapet O, ClosonDejardin MT, Untereiner M, Leduc B, Francois E, Maurel J, Seitz JF, Buecher B, Mackiewicz R, Ducreux M, Bedenne L (2006) Preoperative radiotherapy with or without concurrent fluorouracil and leucovorin in T3-4 rectal cancers: results of FFCD 9203. J Clin Oncol 24:4620-4625

11. Glimelius B (2003) Role of adjuvant chemoradiotherapy for abdominal malignancies. Dig Surg 20:169-179

12. Heald RJ, Ryall RD (1986) Recurrence and survival after total mesorectal excision for rectal cancer. Lancet 1:1479-1482

13. Hermanek P, Hermanek PJ (2000) Role of the surgeon as a variable in the treatment of rectal cancer. Sem Surg Oncol 19:325-329

14. Herschman HR, Xie W, Reddy S (1995) Inflammation, reproduction, cancer and all that.... The regulation and role of the inducible prostaglandin synthase. Bioessays 17:1031-1037 
15. Joo YE, Kim HS, Min SW, Lee WS, Park CH, Park CS, Choi SK, Rew JS, Kim SJ (2002) Expression of cyclooxygenase-2 protein in colorectal carcinomas. Int J Gastrointest Cancer 31:147-154

16. Kapiteijn E, Putter H, van de Velde CJH and cooperative investigators of the Dutch Colorectal Cancer Group (2002) Impact of the introduction and training of total mesorectal excision on recurrence and survival in rectal cancer in the Netherlands. cooperative investigators of the Dutch Colorectal Cancer Group Br J Surgery 89:1142-1149

17. Kapiteijn E, Marijnen CA, Nagtegall ID Putter H, Steup WH, Wiggers T, Rutten HJ, Pahlman L, Glimelius B, van Krieken JH, Leer JW, van de Velde CJ, Dutch Colorectal Cancer Group (2001) Preoperative radiotherapy combined with total mesorectal excision for resectable rectal cancer. N Engl J Med 345:638-646

18. Kim YB, Kim GE, Cho NH, Cho HC, Pyo HR, Lee JD, Park TK, Koom WS, Chun M, Suh CO (2002) Overexpression of cyclooxigenase-2 is associated with a poor prognosis in patients with squamous cell carcinoma of the uterine cervix treated with radiation and concurrent chemotherapy. Cancer 95:531-539

19. Kishi K, Petersen S, Petersen C, Hunter N, Mason K, Masferrer JL, Tofilon PJ, Milas L (2000) Preferential enhancement of tumor radioresponse by an inhibitor of cyclooxygenase-2 inhibitor. Cancer Res 60:1326-1331

20. Konno H, Baba M, Shoji T, Ohta M, Suzuki S, Nakamura S (2002) Cyclooxygenase-2 expression correlates with uPAR levels and is responsible for poor prognosis of colorectal cancer. Clin Exp Metastasis 19:527-534

21. Masferrer JL, Leahy KM, Koki AT, Zweifel BS, Settle SL, Woerner BM, Edwards DA, Flickinger AG, Moore RJ, Seibert K (2000) Antiangiogenic and antitumor activities of cyclooxygenase-2 inhibitors. Cancer Res 60:1306-1311

22. Masunaga R, Kohno H, Dhar DK, Ohno S, Shibakita M, Kinugasa S, Yoshimura H, Tachibana M, Kubota H, Nagasue N (2000) Cyclooxygenase-2 expression correlates with tumor neovascularization and prognosis in human colorectal carcinoma patients. Clin Cancer Res 6:4064-4068

23. Milas L, Kishi K, Hunter N, Mason K (1999) Enhancement of tumor response to gamma-radiation by an inhibitor of cyclooxygenase-2 enzyme. J Natl Cancer Inst 91:1501-1504

24. Milas L (2003) Cyclooxygenase-2 (COX-2) enzyme inhibitors and radiotherapy: preclinical basis. Am J Clin Oncol 26:S66-69

25. Milas L, Mason KA, Crane CH, Liao Z, Masferrer J (2003) Improvement of radiotherapy or chemoradiotherapy by targeting COX-2 enzyme. Oncology 17:15-24

26. Nakata E, Mason KA, Hunter N, Husain A, Raju U, Liao Z, Ang KK, Milas L (2004) Potentiation of tumor response to radiation or chemoradiation by selective cyclooxygenase- 2 enzyme inhibitors. Int J Radiat Oncol Biol Phys 58:369-375

27. Nix P, Lind M, Greenman J, Stafford N, Cawkwell L (2004) Expression of Cox-2 protein in radioresistant laryngeal cancer. Ann Oncol 15:797-801

28. Petersen S, Haroske G, Hellmich G, Ludwig K, Petersen C, Eicheler W (2002) COX-2 expression in rectal carcinoma: immunohistochemical pattern and clinical outcome. Anticancer Res 22:1225-1230

29. Pyo H, Choy H, Amorino GP, Kim JS, Cao Q, Hercules SK, DuBois RN (2001) A selective cyclooxigenase-2 inhibitor, NS398 , enhances the effect of radiation in vitro and in vivo preferentially on the cells that express cyclooxygenase-2. Clin Cancer Res 7:2998-3005

30. Quirke P, Durdey P, Dixon MF, Williams NS (1986) Local recurrence of rectal adeocarcinoma due to inadequate surgical resection: histopathological study of lateral tumor spread and surgical excision. Lancet 2:996-999

31. Sano H, Kawahito Y, Wilder RL, Hashiramoto A, Mukai S, Asai K, Kimura S, Kato H, Kondo M, Hla T (1995) Expression of cyclooxygenase- 1 and-2 in human colorectal cancer. Cancer Res 55:3785-3789

32. Sauer R, Becker H, Hohenberger W, Rödel C, Wittekind C, Fietkau R, Martus P, Tschmelitsch J, Hager E, Hess CF, Karstens JH, Liersch T, Schmidberger H, Raab R (2004) Preoperative versus postoperative chemoradiotherapy for rectal cancer. German Rectal Cancer Study Group N Engl J Med 17:1731-1740

33. Sheehan KM, Sheahan K, O'Donoghue DP, MacSweeney F, Conroy RM, Fitzgerald DJ, Murray FE (1999) The relationship between cyclooxygenase-2 expression and colorectal cancer. JAMA 282:1254-1257

34. Smith WL, Garavito RM, Dewitt DL (1996) Prostaglandin endoperoxide $\mathrm{H}$ synthases (cyclooxygenases)-1 and -2. J Biol Chem 271:33157-33160

35. Sobin LH, Wittekind C (eds) (1997) TNM Classification of malignant tumours. 5th ed. John Wiley \& Sons, New York

36. Soumaoro LT, Uetake H, Higuchi T, Takagi Y, Enomoto M, Sugihara K (2004) Cyclooxygenase-2 expression: a significant prognostic indicator for patients with colorectal cancer. Clin Cancer Res 10:8465-8471

37. Steinauer KK, Gibbs I, Ning S, French JN, Armstrong J, Knox SJ (2000) Radiation induces upregulation of cyclooxygenase-2 (COX-2) protein in PC-3 cells. Int J Radiat Oncol Biol Phys 48:325-328

38. Swedish Rectal Cancer Trial (1997) Improved survival with preoperative radiotherapy in resectable rectal cancer. $\mathrm{N}$ Engl $\mathrm{J}$ Med 336:980-987

39. Terakado N, Shintani S, Yano J, Chunnan L, Mihara M, Nakashiro $\mathrm{K}$, Hamakawa H (2004) Overexpression of cyclooxygenase-2 is associated with radioresistance in oral squamous cell carcinoma. Oral Oncol 40:383-389

40. Tomozawa S, Tsuno NH, Sunami E, Hatano K, Kitayama J, Osada T, Saito S, Tsuruo T, Shibata Y, Nagawa H (2000) Cyclooxygenase-2 overexpression correlates with tumour recurrence, especially haematogenous metastasis, of colorectal cancer. Br J Cancer 83:324-328

41. Tsujii M, Dubois R (1995) Alterations in cellular adhesion and apoptosis in epithelial cells overexpressing prostaglandin endoperoxide synthase-2. Cell 83:493-501

42. Tsujii M, Kawano S, Dubois R (1997) Cyclooxigenase-2 expression in human colon cancer cells increases metastatic potential. Proc Natl Acad Sci U S A 94:3336-3340

43. Tucker ON, Dannenberg AJ, Yang EK, Zhang F, Teng L, Daly JM, Soslow RA, Masferrer JL, Woerner BM, Koki AT, Fahey TJ 3rd (1999) Cyclooxigenase-2 expression is upregulated in human pancreatic cancer. Cancer Res 59:987990

44. Wambura C, Aoyama N, Shirasaka D, Kuroda K, Maekawa S, Ebara S, Watanabe Y, Tamura T, Kasuga M (2002) Effect of Helicobacter pylori-induced cyclooxygenase-2 on gastric epithelial cell kinetics: implication for gastric carcinogenesis. Helicobacter 7:129-138

45. Watwe V, Javle M, Lawrence D, Groth J, Iyer R, El-Hajjar D, Geradts J (2005) Cyclooxygenase-2 (COX-2) levels before and after chemotherapy: a study in rectal cancer. Am J Clin Oncol 28:560-564

46. Williams CS, Mann M, Dubois RN (1999) The role of cyclooxygenases in inflammation, cancer, and development. Oncogene 18:7908-7916

47. World Health Organization (1988) International histological classification of tumours. 2nd ed. Geneva: World Health Organization, 1969-1981. Springer-Verlag, Berlin

48. Zhang H, Sun XF (2002) Overexpression of cyclooxygenase-2 correlates with advanced stages of colorectal cancer. Am J Gastroenterol 97:1037-1041 